\title{
Brain network efficiency is influenced by the pathologic source of corticobasal syndrome OPEN
}

John D. Medaglia, PhD Weiyu Huang Santiago Segarra, PhD Christopher Olm James Gee, PhD Murray Grossman, MD Alejandro Ribeiro, PhD Corey T. McMillan, PhD Danielle S. Bassett, PhD

Correspondence to Dr. Bassett: dsb@seas.upenn.edu

\section{Supplemental data} at Neurology.org

\section{ABSTRACT}

Objective: To apply network-based statistics to diffusion-weighted imaging tractography data and detect Alzheimer disease vs non-Alzheimer degeneration in the context of corticobasal syndrome.

Methods: In a cross-sectional design, pathology was confirmed by autopsy or a pathologically validated CSF total tau-to- $\beta$-amyloid ratio (T-tau/A $\beta$ ). Using structural MRI data, we identify association areas in fronto-temporo-parietal cortex with reduced gray matter density in corticobasal syndrome $(n=40)$ relative to age-matched controls $(n=40)$. Using these fronto-temporo-parietal regions of interest, we construct structural brain networks in clinically similar subgroups of individuals with Alzheimer disease $(n=21)$ or non-Alzheimer pathology $(n=19)$ by linking these regions by the number of white matter streamlines identified in a deterministic tractography analysis of diffusion tensor imaging data. We characterize these structural networks using 5 graph-based statistics, and assess their relative utility in classifying underlying pathology with leave-one-out cross-validation using a supervised support vector machine.

Results: Gray matter density poorly discriminates between Alzheimer disease and non-Alzheimer pathology subgroups with low sensitivity (57\%) and specificity (52\%). In contrast, a statistic of local network efficiency demonstrates very good discriminatory power, with $85 \%$ sensitivity and $84 \%$ specificity.

Conclusions: Our results indicate that the underlying pathologic sources of corticobasal syndrome can be classified more accurately using graph theoretical statistics derived from patterns of white matter network organization in association cortex than by regional gray matter density alone. These results highlight the importance of a multimodal neuroimaging approach to diagnostic analyses of corticobasal syndrome. Neurology ${ }^{\circledR}$ 2017;89:1373-1381

\section{GLOSSARY}

$\mathbf{a A D}=$ amnestic Alzheimer disease; $\mathbf{A D}=$ Alzheimer disease; $\mathbf{C B S}=$ corticobasal syndrome; $\mathbf{F A}$ = fractional anisotropy.

Two distinct neuropathologies account for the majority of causes of corticobasal syndrome (CBS): (1) a form of tauopathy associated with the accumulation of misfolded and hyperphosphorylated tau or (2) Alzheimer disease (AD) with deposits of $\beta$-amyloid $(A \beta)$ in the form of neuritic plaques and paired helical filaments of tau that result in neurofibrillary tangles. ${ }^{1}$ At present, the pathology of underlying CBS is predicted antemortem in only $25 \%-56 \%$ of cases. ${ }^{1}$

Recent clinically heterogeneous studies have demonstrated that white and gray matter MRI measurements can help distinguish $\mathrm{AD}$ from non-Alzheimer pathologies such as tauopathy, ${ }^{2,3}$ but may have been confounded by heterogeneous patterns of disease distribution associated with clinical phenotypes rather than underlying pathologies. More recently, it has been demonstrated that with $\mathrm{CBS}$ there is a dissociation of greater gray matter disease associated with $\mathrm{AD}$ pathology

\footnotetext{
From the Departments of Psychology (J.D.M.), Electrical and Systems Engineering (W.H., D.S.B.), Neurology (C.O., M.G., C.T.M.), and Bioengineering (D.S.B.), Penn Frontotemporal Degeneration Center (C.O., M.G., C.T.M.), and Penn Image Computing and Science Lab (J.G.), University of Pennsylvania, Philadelphia; and Institute for Data, Systems, and Society (S.S., A.R.), Massachusetts Institute of Technology, Cambridge.

Go to Neurology.org for full disclosures. Funding information and disclosures deemed relevant by the authors, if any, are provided at the end of the article. The Article Processing Charge was funded by the NIH.

This is an open access article distributed under the terms of the Creative Commons Attribution-NonCommercial-NoDerivatives License 4.0 (CC BY-NC-ND), which permits downloading and sharing the work provided it is properly cited. The work cannot be changed in any way or used commercially without permission from the journal.
} 


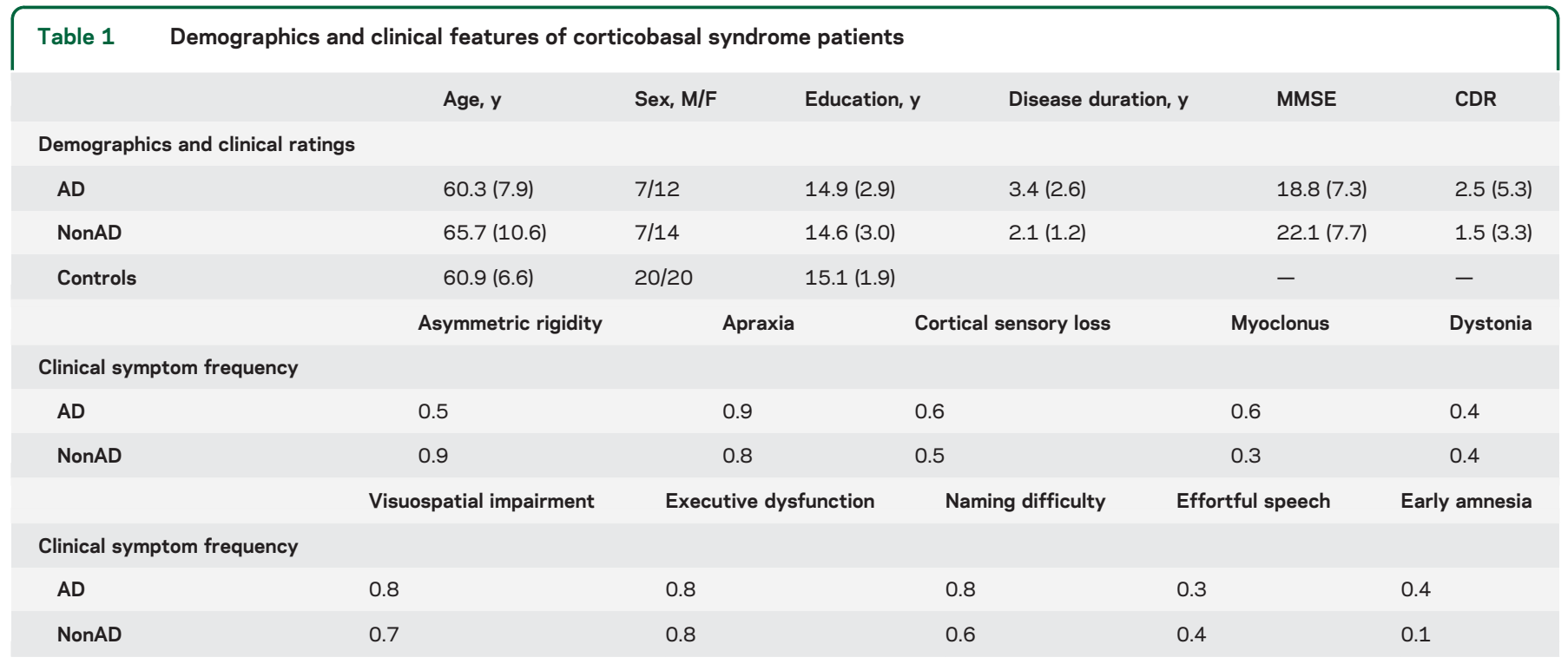

Abbreviations: $A D=$ Alzheimer disease; $C D R=$ Clinical Dementia Rating; MMSE = Mini-Mental State Examination.

Demographic, CDR, and MMSE information is presented as mean (SD). Values for CDR are median (interquartile range). Clinical features are presented as the proportions of observed features noted on patient charts in the current sample. Classification accuracy was tested for robustness against demographic and neuropsychological symptoms. See "Relationship of network classification outcomes to extrinsic variables" in the supplemental data for a discussion of misclassifications.

and greater white matter disease associated with non-Alzheimer, or likely frontotemporal dementia, pathology. ${ }^{4}$ However, this prior evidence has not considered classification of individual patients or network features.

Network science offers methods to analyze complex relational data, and has increasingly been applied to neuroimaging data. Recent evidence suggests that network hubs in fronto-temporo-parietal systems play critical roles in normative processes associated with cognitive and motor function ${ }^{5}$ and that their failure leads to clinical abnormalities. ${ }^{6}$ Yet, specific neuropathologic mechanisms linking network properties to observable behavioral phenotypes in clinical disorders have remained elusive.

Here, we address these prior gaps by applying a network analytic technique to multimodal neuroimaging data to differentiate pathologic drivers of CBS. We hypothesize that $\mathrm{AD}$ pathology and non-Alzheimer pathology such as tauopathy may contribute to subtle differences in the degeneration of frontotemporo-parietal regions-key loci of multiple cognitive and motor hubs implicated in CBS - and that this is discernible as differential network patterns. Specifically, we expect widespread gray matter density reductions in frontal, parietal, and temporal lobes in patients with CBS, regardless of the specific pathology. ${ }^{7}$ We further anticipate that network characteristics associated with areas of reduced gray matter density in CBS display discriminable patterns in non-Alzheimer vs $\mathrm{AD}$ pathology, representing complex pathologic consequences of these diseases. We test these predictions in a clinically homogeneous population with CBS using diffusion-weighted images in participants with non-Alzheimer or $\mathrm{AD}$ pathology confirmed by autopsy or autopsy-validated CSF tau/A $\beta$ ratios.

METHODS Participants. The patient cohort included 40 individuals from the Penn Frontotemporal Degeneration Center and Cognitive Neurology Clinic at the University of Pennsylvania who were clinically diagnosed with CBS and 40 age- and sex-matched elderly controls. A board-certified neurologist with extensive expertise in neurodegenerative diseases diagnosed all patients using published criteria. ${ }^{1} \mathrm{AD}$ or nonAD pathology was confirmed by autopsy, genetic screen, or CSF analysis (see CSF analysis and figure e- 1 at Neurology. org). Throughout the rest of the article, we consider 3 groups of interest: healthy elderly controls, individuals with $\mathrm{AD}$ pathology presenting as $\mathrm{CBS}$, and individuals with nonAD pathology presenting as CBS confirmed by autopsy, genetics, or $\mathrm{T}$-tau/A $\beta$ ratio. We refer to these groups as controls, CBS due to AD (CBS-AD), and $\mathrm{CBS}$ due to non-Alzheimer pathology (CBS-nonAD), respectively. In all neuroimaging analyses including machine learning approaches, tests were applied to identify differences among these group designations. Patient groups were comparable on education and overall disease severity measured with the Mini-Mental State Examination and Clinical Dementia Rating scale (all $p>0.1$ ). Disease duration was significantly different $(p=0.04)$ between groups (see e-Results, "Relationship of network classification outcomes to extrinsic variables," concerning the robustness of results to 
Figure 1 Schematic of the method
A
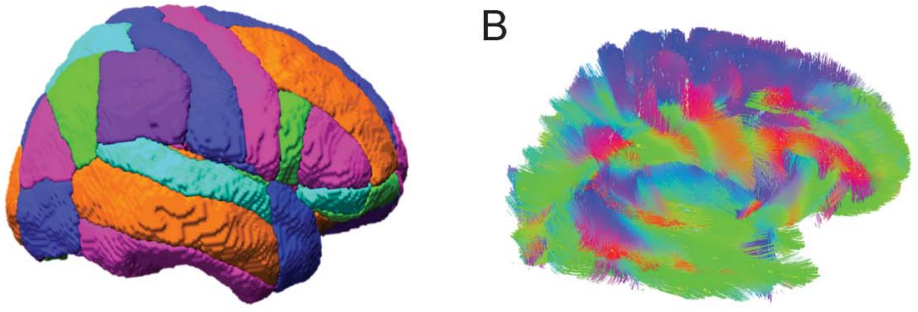

C

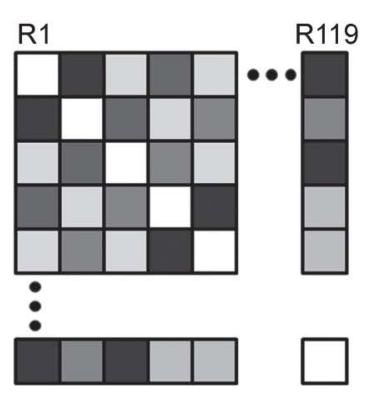

D

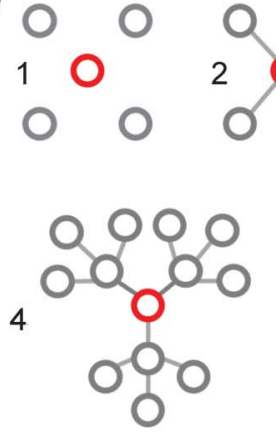

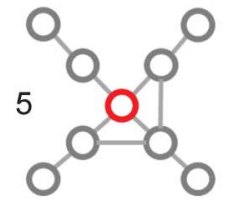

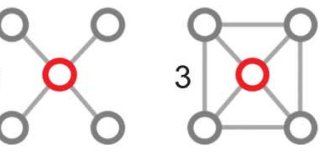

$E$

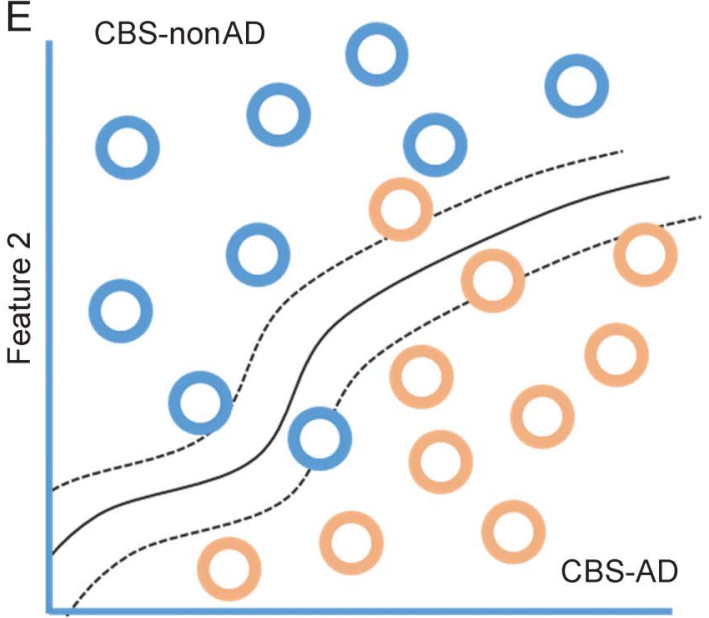

Feature 1

(A) Regions of interest $(\mathrm{n}=119)$ were defined by OASIS labels registered to each individual's structural T1. (B) For each individual, we performed diffusion tractography to estimate streamlines connecting all voxel pairs. (C) $\mathrm{An} \mathrm{N} \times \mathrm{N}$ adjacency matrix $\mathrm{A}$ whose element Aij represents the number of streamlines reconstructed between region $\mathrm{i}$ and region $\mathrm{j}$. We refer to each region as a network node, and each region-to-region connection as a network edge, weighted by the number of connecting streamlines. (D) We used 5 statistics at each node to classify non-Alzheimer vs Alzheimer disease (AD): (1) gray matter density, which is agnostic to network connectivity; (2) node strength, which represents total edge weight of connections incident to or equivalently emanating from a region; (3) local clustering, which represents the extent to which a node's neighbors are also connected to each other; (4) eigenvector centrality, which is a statistic for the overall influence of a node in a network; and (5) local efficiency, which offers a measurement characterizing how connected the neighbors of a node are when this node is deleted. Note that in this illustration, the local efficiency for the node of interest is low. (E) Using the 5 statistics illustrated pictorially in panel $D$, we apply a support vector machine to training data (see "support vector machine analysis") to determine the classification measures and performance, equally weighted in sensitivity and specificity. $\mathrm{CBS}=$ corticobasal syndrome.

variables of noninterest). Patients were also matched on clinician evaluation of the frequency of asymmetric rigidity, apraxia, cortical sensory loss, myoclonus, dystonia, visuospatial impairments, executive dysfunction, naming difficulty, and effortful speech (table 1).
Standard protocol approvals, registrations, and patient consents. Written informed consent was obtained from all study participants using a protocol approved by an institutional review board convened at the University of Pennsylvania.

Neuroimaging methods. See the supplemental data for a description of our neuroimaging acquisition and preliminary processing methods.

Network methods. See figure 1 for a schematic and description of methods used to construct structural networks and train classifiers. See the supplemental data for further discussion and mathematical definitions of network features.

Network construction and regional statistics. Network science is a framework for representing and analyzing complex relational data. ${ }^{8}$ In this framework, components of a system are referred to as nodes, and connections between nodes are referred to as edges. Together, the nodes and the edges that connect them form a graph, which can be studied using techniques developed in the field of mathematics known as graph theory. A graph can be summarized in the form of an $\mathrm{N} \times \mathrm{N}$ adjacency matrix A. Here, we generated an adjacency matrix of size $119 \times 119$ for each participant. Each matrix element gives the number of streamlines connecting region $i$ with region $j$. Using each individual's adjacency matrix, we calculated 5 commonly applied network statistics at each of the 119 brain regions. We selected the statistics based on the representation in the literature and theoretical relevance in their putative roles in mediating network dynamics. Specifically, we examined (1) strength (sometimes referred to as "weighted degree," here defined as the sum of streamline counts to that particular region), (2) strength corrected for total edge weight in the network (also known as network density), (3) local clustering coefficient, (4) eigenvector centrality, and (5) local efficiency. See the supplemental data for mathematical definitions and reference 9 for a discussion regarding the usage of network statistics in neuroimaging data.

Support vector machine analysis. Support vector machine is a supervised learning method for binary classification, ${ }^{10}$ and is therefore often used to classify observations (e.g., patients) into 2 possible classes (e.g., CBS-nonAD and CBS-AD). We treat the following 3 types of data as features: gray matter density, white matter streamline counts connecting pairs of regions, and network statistics. We then train the support vector machines by providing them with labeled observations, for which the classification results are known. To overcome limitations evident in the use of linear classifiers, we employ nonlinear classification using kernels, ${ }^{11}$ which transform the feature space such that a linear classifier trained in the kernelized space is a nonlinear classifier in the original feature space (figure 1E for an illustration of a nonlinear classifier).

Classifier training and testing. We evaluate classification power for gray matter density and network statistics calculated from cortical labels that displayed significantly reduced gray matter density in CBS (both CBS-AD and nonCBS-nonAD) in comparison to healthy controls. We assign a label (CBS-nonAD or CBS-AD) to each observation (patient in the CBS cohort) based on that patient's likely pathology determined using autopsy, genetic, or CSF $\mathrm{T}$-tau/A $\beta$ ratio. We utilized labeled features for all observations except one to train a support vector machine classifier and compared the pathology predicted by the classifier for the left-out observation with his or her actual pathology. ${ }^{12}$ This process was repeated 40 times (i.e., the number of individuals in the clinical sample), each time with a different individual excluded from the training phase. We defined the 
Figure 2 Gray matter differences between corticobasal syndrome and healthy controls, and between Alzheimer disease (AD) and nonAD pathology

A

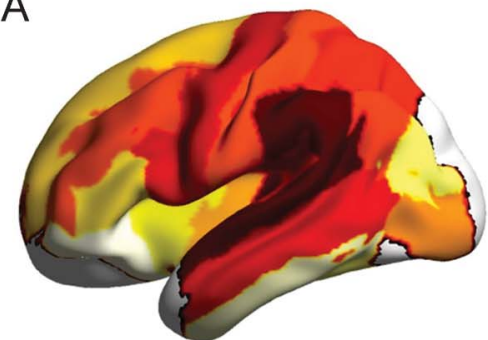

L
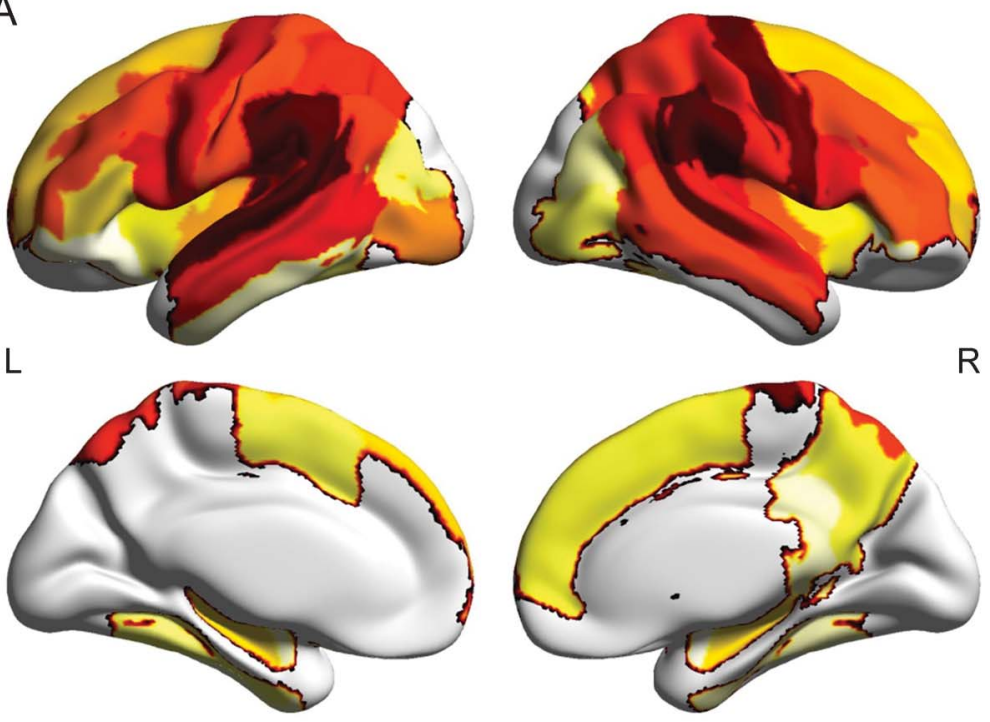

$\mathrm{R}$

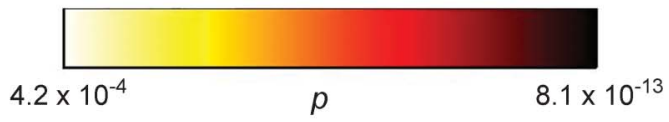

B

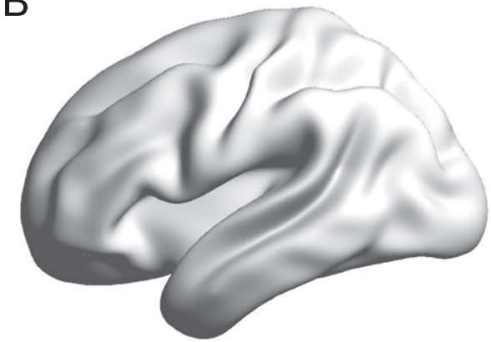

L
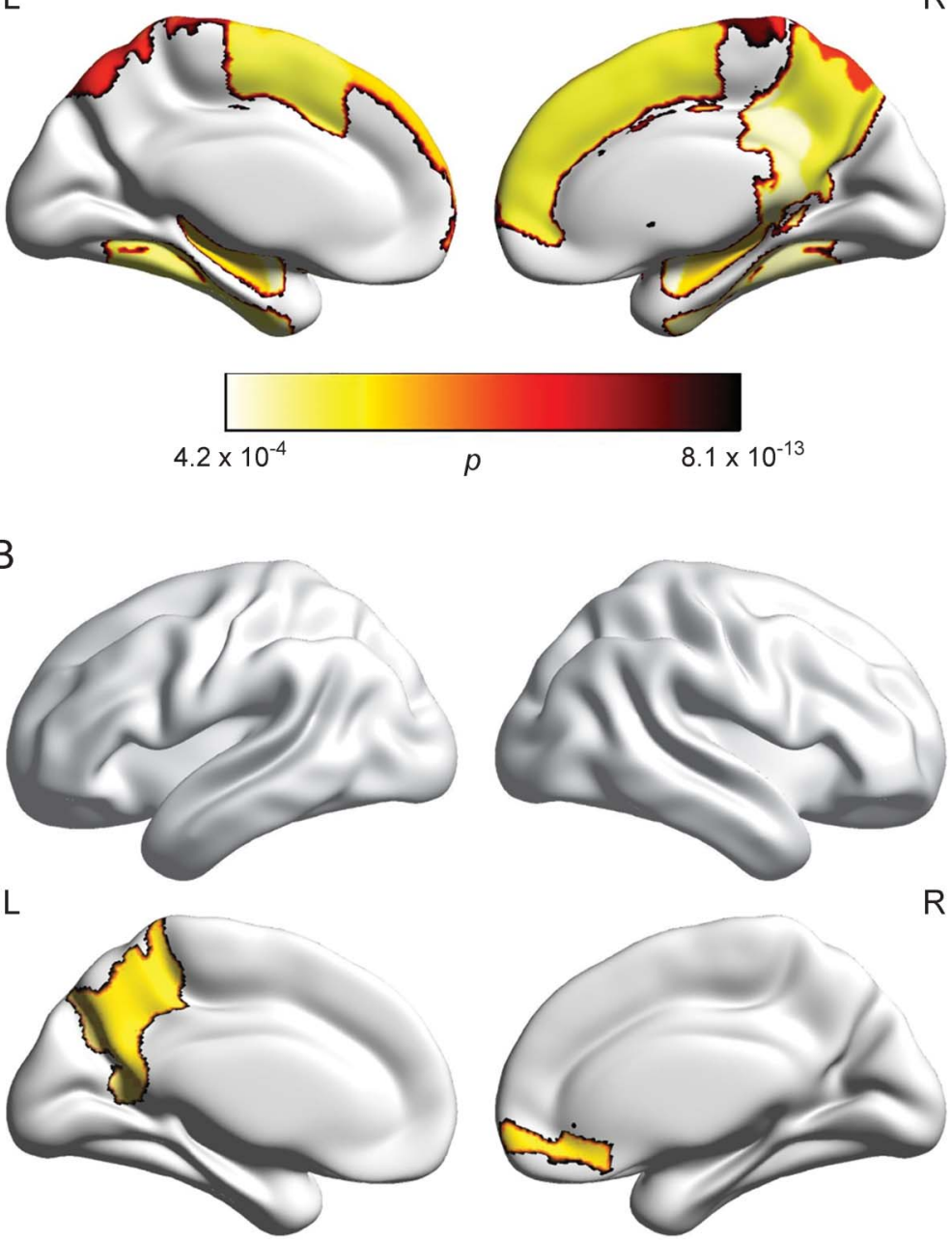

$8.1 \times 10^{-13}$

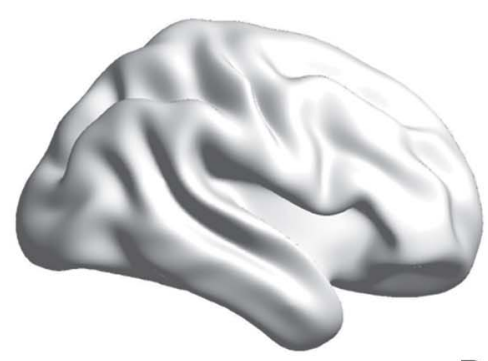

$\mathrm{R}$

(A) Regions with significantly reduced gray matter density in individuals with corticobasal syndrome relative to controls following Bonferroni correction for multiple comparisons. A wide range of regions within the frontal, parietal, and temporal lobes demonstrated reduced density in the diseased cohort. (B) Regions with significantly different gray matter density in individuals with nonAD relative to $A D$. In all cases, volumes were reduced in $A D$ relative to nonAD. Observe that only one region in the left precuneus and one region in the right medial frontal gyrus are found to be statistically significant following Bonferroni correction for multiple comparisons. In both panels, hotter colors indicate increasing statistical significance.

global sensitivity and specificity of the classification to be the average performance over all trials for each feature. See the supplemental data for additional details.

Majority vote. Each network statistic can be sensitive to finescale differences in pathologic drivers. Pragmatically, we may wish to combine information from all statistics to maximize sensitivity to individual differences. Combined classifiers generally lead to better classification results by including more diverse information. ${ }^{13}$ In our "majority vote" classifier, each individual was assigned to either the predicted CBS-nonAD group or the predicted CBS-AD group according to the most frequently predicted assignment across the 5 network statistics.

RESULTS Gray matter density as a disease biomarker. To test the hypothesis that the gray matter in frontal, temporal, and parietal cortex would be more affected in CBS compared to healthy individuals, we tested for regional differences in gray matter density using 2tailed $t$ tests comparing the gray matter values from controls to the joint sample of CBS-AD and CBSnonAD pathology. We applied a Bonferroni correction $^{13}$ for multiple comparisons based on an $\alpha$ value of 0.05 (corrected value: $p=0.0004$ ) and observed that 62 of the 119 regions displayed significantly less gray matter density in CBS in comparison to controls. These regions were anatomically located over a broad distribution of bilateral fronto-temporoparietal cortex, including the primary and supplementary motor cortices, as well as the bilateral insula (figure 2 and table e-1). We use these 62 areas as regions of interest in the following classification analysis.

White matter streamline differences as disease biomarkers. To contextualize our machine learning approach applied to network statistics, we tested for differences in the number of streamlines connecting pairs of regions in (1) elderly individuals compared to those with CBS (CBS-AD and nonCBS-nonAD pathology) in addition to (2) individuals with CBSnonAD relative to CBS-AD. We applied a Bonferroni correction ${ }^{14}$ for multiple comparisons assuming an $\alpha$ level of 0.05 (corrected value: $p=3.5 \times 10^{-6}$ ) and observed that streamlines connecting 9 pairs of regions were reduced in CBS relative to controls predominantly within and between the right frontotemporal cortex, in addition to a pair in the left frontal cortex and one interhemispheric connection between the left medial frontal cortex and right medial superior frontal gyrus (figure 3 and table e-2). There were no region pairs with significantly different streamline counts between individuals with CBSnonAD and $\mathrm{AD}$. See the supplemental data for a list of all regions with a reduced number of streamlines in CBS relative to controls and a discussion of our results compared to voxelwise measures of white matter integrity in CBS. ${ }^{4}$

Network statistics as a pathology biomarker: A machine learning approach. We trained support vector machines to identify nonCBS-AD relative to $\mathrm{AD}$ pathology on each of the 5 network statistics calculated for the 62 regions displaying reduced gray matter volume in CBS (see also the supplemental data for analyses comparing CBS-AD and CBS-nonAD 

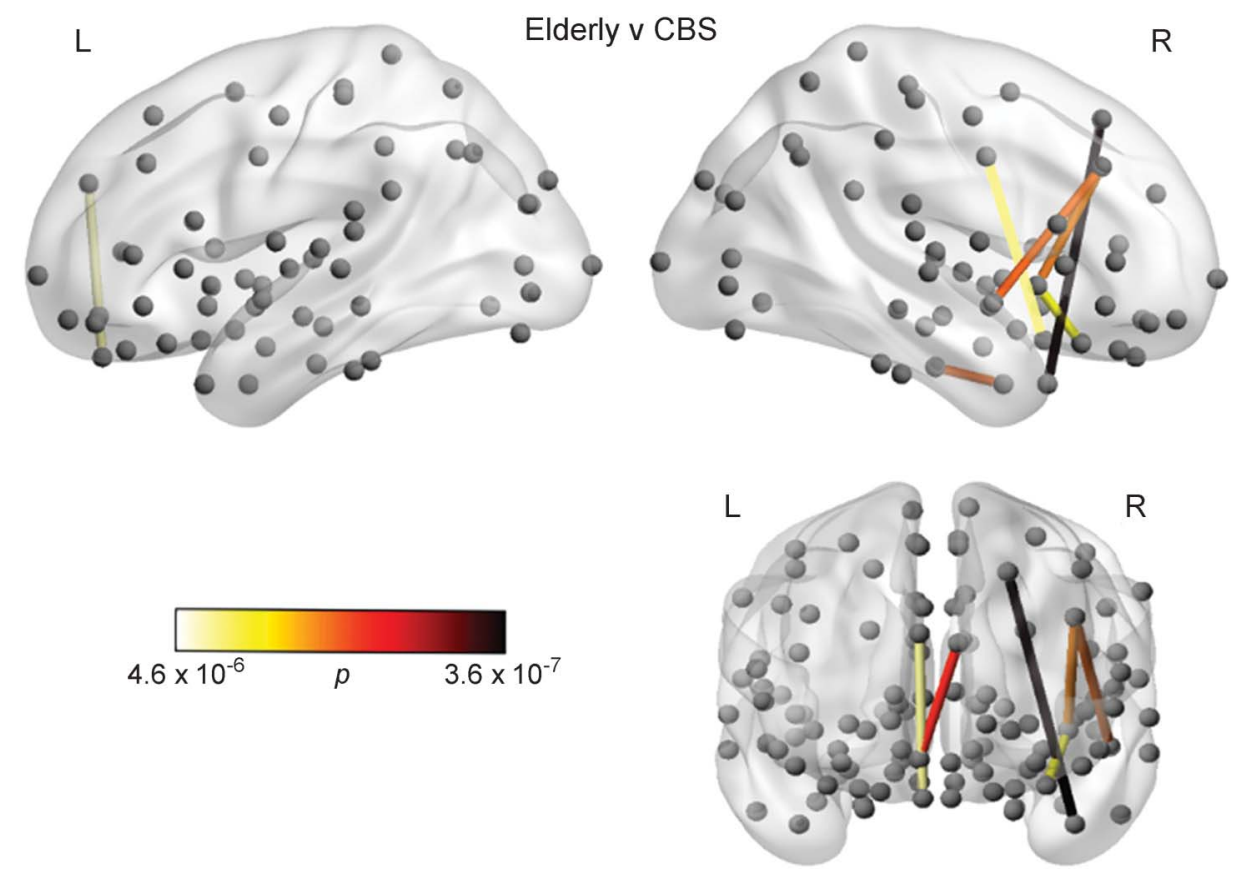

Region pairs with significantly reduced white matter streamline counts in individuals with CBS relative to controls. Differences were most prominently observed in the right frontal and temporal cortices. Coronal representation is viewed facing the anterior surface. Hotter colors for connections indicate increasing statistical significance. See the supplemental data for a list of region pairs with a reduced number of streamlines.

to an amnestic $\mathrm{AD}$ and amnesic mild cognitive impairment sample). We observed the best classification performance when using the local efficiency of regions as the features in the support vector machine: performance reached a peak sensitivity of $85 \%$ and a peak specificity of $84 \%$ (see figure e- 2 for results across classifier parameters). Other network statistics offered more modest sensitivities and specificities compared to local efficiency. While local efficiency reflects all areas of gray matter atrophy in CBS, the regions contributing the highest weights to classification performance include left middle temporal gyrus, right temporal-parietal-occipital cortex, and bilateral insula (figure 4 and figure e-3; see also figure e-4 representing the relationship between local efficiency and $\mathrm{T}$-tau/A $\beta$ ratios).

To determine the relative utility of network statistics in comparison to univariate descriptors in identifying CBS-nonAD relative to CBS-AD pathology, we trained and tested support vector machines using either (1) gray matter density values or (2) all white matter streamlines connecting pairs of regions within the 62 regions shown to have reduced gray matter density relative to the healthy control sample. The classifier based on regional gray matter density offered a maximum sensitivity of $57 \%$ and a maximum specificity of $52 \%$. These results indicate that gray matter density measurements do not strongly distinguish between the 2 pathologies underlying CBS. We next examined differences in peak performance (maximum shared sensitivity/specificity) between network and non-network statistics. ${ }^{15}$ Only local efficiency demonstrated statistically superior performance to that observed when using gray matter density ( $84 \%$ vs $52 \%$; $\left.\chi^{2}[1]=7.26 ; p=0.035\right)$ and to that observed when using white matter streamline counts $(84 \%$ vs $52 \%$; $\left.\chi^{2}[1]=7.26 ; p=0.035\right)$. Note that $p$ values are corrected for multiple comparisons within the gray matter and white matter comparisons separately due to their methodologically independent source.

Three other network statistics demonstrated statistically significant classification performance relative to chance $(50 \%$ classification) as determined using a binomial sign test: strength $(p=0.008)$, strength corrected for network density ( $p=0.003$ ), and eigenvector centrality $(p=0.003)$. See figure e-5 for peak sensitivity and specificities for each tested neuroimaging measure. We also evaluated a decision tree approach to network statistic-based classification to examine the relative utility of the support vector machine approach, but this did not yield any statistically significant effects (see figure e-6).

The majority vote across network statistics offered comparable performance to that obtained from the local efficiency alone (against gray matter: $84 \%$ vs $52 \% ; \chi^{2}[1]=7.26 ; p=0.007$; against white matter: $84 \%$ vs $\left.52 \% \chi^{2}[1]=7.26 ; p=0.007\right)$. Collectively, these results indicate that the 5 selected network 
Figure 4 Discriminating power of different feature sets

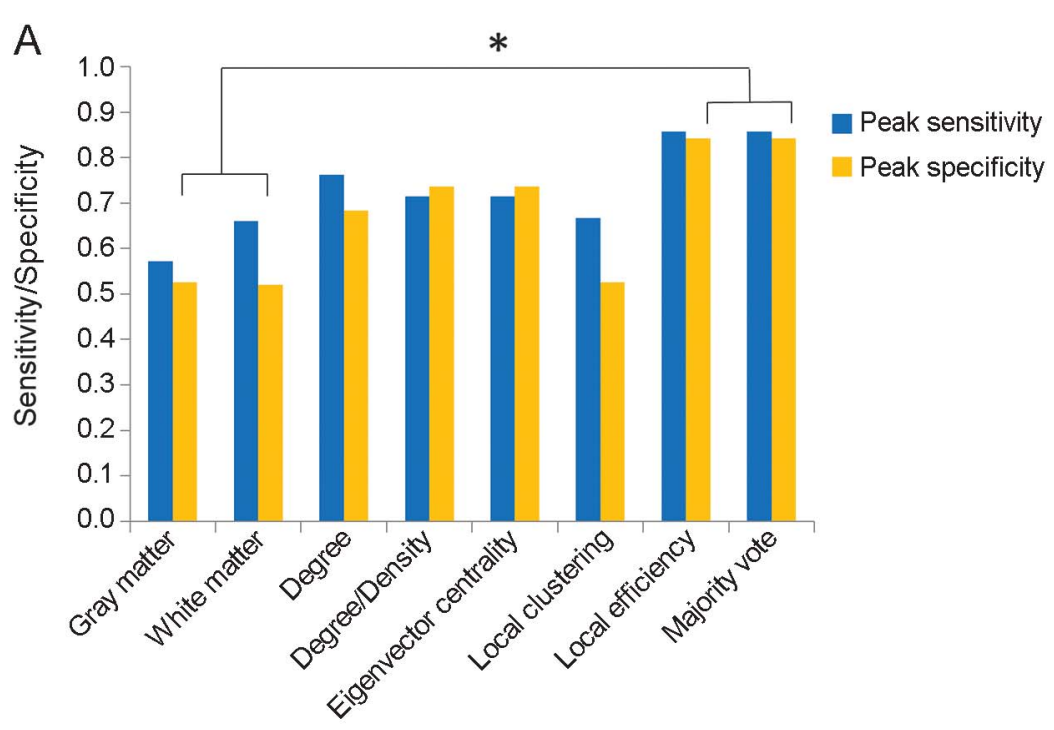

B
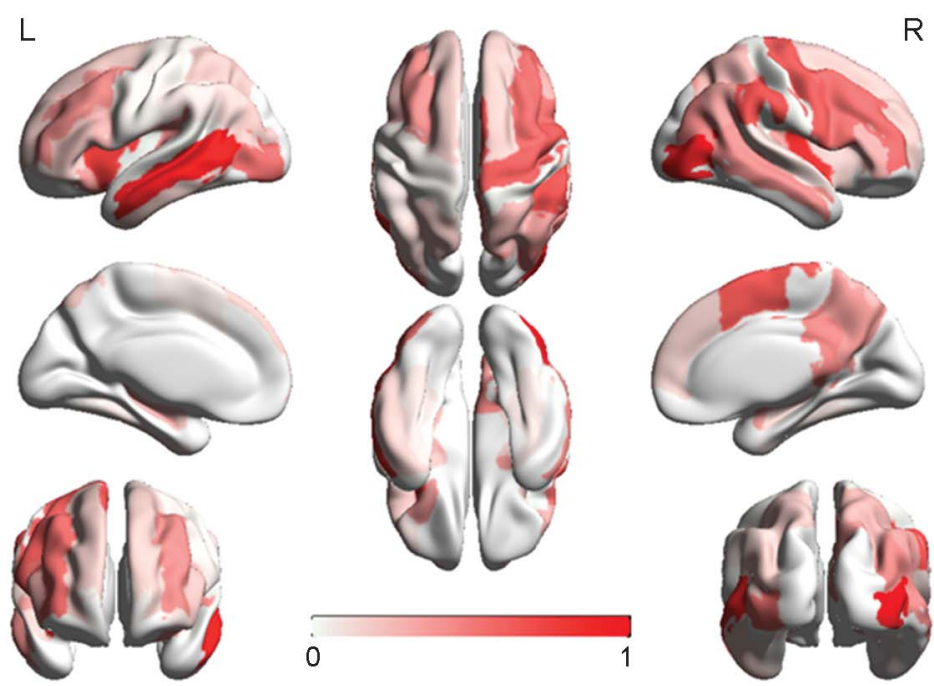

Sensitivity (blue) and specificity (yellow) for gray matter density and white matter streamlines (left), network statistics (middle), and majority vote (right). (A) Asterisk denotes that local efficiency and the majority vote perform significantly better than gray matter and white matter streamline classifiers. Observe that both sensitivity and specificity demonstrate approximately chance performance when only regional gray matter density is considered. (B) The spatial distribution of weights for the local efficiency classifier. In this classifier, the left middle temporal gyrus, bilateral insula, and right lateral temporal-parietal-occipital regions contribute the highest weight. Importantly, this weight is assigned in the context of the entire support vector machine (SVM) classifier; thus, the weights are only meaningful in the context of all regions contributing to the classifier. Increasing red represents increasing absolute values of normalized weight in the classifier on a (0:1) interval.

statistics robustly classify CBS-nonAD from CBS$\mathrm{AD}$, but that local efficiency drives most of the classifier performance (see figure 4 and table e-3; see "Relationship of network classification outcomes to extrinsic variables" in the supplemental data for a discussion of misclassifications).

We also assessed the discriminating value of global efficiency (at a conceptual level, a measure that is inversely related to the path length) across the entire network. While this measure discriminated between all patients and controls with $82.5 \%$ accuracy (minimum sensitivity/specificity $=80 \%$ ), we found that global efficiency does not discriminate between CBS$\mathrm{AD}$ and CBS-nonAD patient groups (accuracy = $65 \%$; minimum sensitivity/specificity $=58 \%)($ see e-Results, also for a model associating CSF T-tau/ $A \beta$ ratios with white matter local efficiency values).

Finally, we examined a cohort of 20 demographically and severity-matched patients with amnestic mild cognitive impairment and amnestic $\mathrm{AD}$ $(\mathrm{ADD})$, and found that local efficiency robustly discriminates between CBS-nonAD patients and $\mathrm{aAD}$ patients (accuracy $=100 \%$; minimum sensitivity/ specificity $=100 \%$ ). In addition, local efficiency robustly discriminates between CBS-AD and $\mathrm{aAD}$ patients (accuracy $=92.3 \%$; minimum sensitivity/ specificity $=90 \%$; see e-Results "Additional clinical cohort: amnestic Alzheimer and mild cognitive impairment" section).

DISCUSSION Our results demonstrate that network techniques applied to white matter tractography can provide high accuracy in classifying the underlying neuropathology contributing to CBS. Anatomical network features can offer unique diagnostic value relative to other traditional neuroimaging measures in samples (CBS-AD and CBS-nonAD) where classification via clinical characteristics or neuroimaging is particularly challenging. The underlying neuropathology is captured most strongly by "local efficiency," which offers an intuitive measure of the robustness of a local network to the degradation of a gray matter node. These findings demonstrate that multimodal imaging, network science, and machine learning can be used to quantify distinct pathologies underlying clinically homogeneous samples. ${ }^{3}$

We found broad fronto-temporo-parietal gray matter loss in CBS due to CBS-AD or CBS-nonAD pathology. ${ }^{6}$ However, evaluation of gray matter loss alone did not discriminate effectively between CBSnonAD and CBS-AD pathology. Specifically, we found $\mathrm{AD}$-specific gray matter disease in the precuneus relative to healthy adults, consistent with findings in $\mathrm{AD}$ not presenting as $\mathrm{CBS} .{ }^{16}$ This brain area is often associated with visual attention, the alteration of which is a clinical characteristic of CBS. We also found significant gray matter atrophy in ventral medial frontal cortex in CBS-AD relative to CBSnonAD. While this medial frontal area is often noted as the focus of disease in patients with a behavioral variant of frontotemporal degeneration, ${ }^{17}$ and while some patients with pathologically confirmed corticobasal degeneration may have disease in this region, ${ }^{18}$ this area is associated with a disorder of personality and social cognition, and most patients participating in this study did not have prominent changes in 
personality and cognition. Regardless of the clinical consequences of disease in these regions, the analysis of patterns of gray matter change in frontal, temporal, and parietal regions affected by CBS was not able to classify the corticobasal patients participating in this study on the basis of their underlying pathology.

Within regions displaying reduced gray matter density in CBS, the classification power of local efficiency was superior to that obtained from white matter streamlines connecting pairs of regions or from regional gray matter density. Local efficiency in certain frontal, temporal, and parietal regions contributed the highest weights to the discrimination between CBS-nonAD and CBS-AD pathology, while global efficiency of the gray matter reduced network was not as effective at discriminating between these underlying pathologies. The performance of local efficiency was similar to a majority vote including the prediction of all 5 network statistics, suggesting that other characteristics of white matter network topology did not substantially contribute critical information sensitive to the pathologic basis for CBS. The strategy applied here is an alternative to feature selection strategies designed to reduce feature redundancy applied in other network analyses. ${ }^{19}$ These strategies typically begin feature selection by examining the correlation structure between features and group assignments. However, this may result in circular feature selection within a given sample. The current work demonstrates that network feature selection can be informed by independently calculated measures of gray matter degeneration prior to training classifiers. This strategy could be examined in the context of other neurodegenerative conditions to identify robust structural network classifiers.

Importantly, prior work has demonstrated that voxel and region-wise white matter analysis of fractional anisotropy (FA) values can discriminate between CBS-AD and CBS-nonAD pathology. ${ }^{4}$ Here, we demonstrate that the differential consequences of $\mathrm{AD}$ and nonAD pathology are not strongly discriminable when examining the number of estimated streamlines. Streamlines are constructed from paths through the principal directions of voxel orientations, whereas FA quantifies the degree of directedness of individual voxels regardless of their direction. Here, we demonstrate that complex network topology can discriminate CBS-AD and CBSnonAD pathology, and can additionally identify amnestic $\mathrm{AD}$ and mild cognitive impairment relative to both CBS-AD and CBS-nonAD groups. Considered together, the current results suggest that in addition to sensitivity to pathology in the overall fractional anisotropy of white matter, ${ }^{4}$ pathologic expressions in structural white matter topology differ significantly across these groups.
Our findings extend prior theories positing that network-based statistics of neuroimaging data can provide unique diagnostic value in neurologic samples. ${ }^{20}$ Pathologic burdens to the brain may appear similar in gross neuroimaging approaches that apply univariate techniques, but may have dissociable effects on network topology. It is particularly informative in the study of patients with CBS because of the differences in white matter disease in the CBS-AD and CBS-nonAD pathologies implicated in CBS. ${ }^{2}$ Our work demonstrates detectably different patterns of network failure in the brain despite similar clinical presentations. Widely distributed atrophy in frontotemporo-parietal gray matter characterizes CBS compared to matched elderly controls, and clinical features in this sample may result from similar effects on local gray matter degeneration due to various histopathologic abnormalities that are insensitive to the simple anatomical distribution of disease.

Our findings suggest that advanced analytic methods may be more sensitive to disease mechanisms reflected by dissociable local efficiency patterns in the Alzheimer and non-Alzheimer groups. In $\mathrm{AD}$, white matter pathology is primarily due to Wallerian degeneration that follows from gray matter disease. Wallerian degeneration is also evident in non $\mathrm{AD}$ pathology including frontotemporal lobar degeneration spectrum pathology. The form of frontotemporal lobar degeneration associated specifically with tau pathology, the other major cause of CBS presumed to be present in our non-Alzheimer group, ${ }^{1}$ is also uniquely associated with specific white matter disease such as astrocytic plaques. ${ }^{21}$ The current findings suggest that the net effect of differences in white matter pathology at the network level result in detectable differences in network organization between Alzheimer and non-Alzheimer-related CBS. Future studies could examine this hypothesis by using postmortem pathology and by linking genetic variants to patterns of structural network degeneration.

Network approaches can additionally enlighten us as to the nature of complex network failures underlying cognitive dysfunction, ${ }^{6}$ perhaps resulting from damage to cognitive "hubs" that support a broad set of functions. ${ }^{22}$ Here, local efficiency is particularly interesting when considering cognitive resilience. This statistic measures the local tolerance of the network to a node's removal and is thought to describe the importance of a node in information transfer in subnetworks. ${ }^{23}$ This is because local efficiency can be thought to represent the degree to which neighbors of a node communicate with one another via short paths. Thus, this measure represents a notion of robustness in the context of neurologic syndromes ${ }^{24}$ : reduced local efficiency at a given node means that its neighbors depend on the node's role for short paths of information transfer in the local neighborhood. If a subnetwork involves many 
nodes with low local efficiency, relatively minor perturbations could have drastic consequences for subnetwork function. Our results suggest that this could have differential consequences for CBS-AD and CBSnonAD pathology: as structural network failures evolve over time, clinical differences may become more salient and produce cognitive and behavioral effects detectable with neuropsychological testing.

Finally, the nosologic status of CBS is a complex behavioral expression of neurobiological damage. CBS is one of 4 clinical phenotypes resulting from corticobasal degeneration, but its nosologic status remains questionable. ${ }^{1}$ We focused on this phenotype and identified underlying differences in neuropathologic effects on network organization. An important goal in CBS and neurologic disorders at large is to characterize disorders from genetic to cognitive-behavioral levels. In corticobasal degeneration, it remains to be seen whether different pathologic and clinical expressions can be linked to distinct network phenotypes more generally. For example, in our CBS-nonAD group, we presume that tau- and TDP-43 pathology contributes to the degeneration that dissociates against CBS-AD pathology. ${ }^{1}$ However, the spectrum of tauopathies is particularly unexplored across neurodegenerative disorders. ${ }^{25}$ It is possible that distinct genetic causes of tauopathy express dissociable network damage and different clinical phenotypes. Our study suggests that linking validated CSF biomarkers with network analysis could be constructive. In the future, similar work that links biomarkers including CSF and genetics with structural network and neuropsychological measurements may provide an important new dimension in clarifying syndrome nosology.

Some limitations apply to this work. First, only a subset of commonly applied network statistics was examined; other statistics may offer additional insights. ${ }^{26}$ Second, we employed deterministic tractography to diffusion imaging data, and defined network edges as the number of streamlines connecting 2 regions $^{27}$; other algorithms and probabilistic approaches may emphasize different features of white matter network organization in CBS. ${ }^{28}$ Third, diffusion imaging data with greater resolution of diffusion directions may increase classification accuracy. There is also the possibility that a small proportion of participants were incorrectly identified as $\mathrm{AD}$ or non $\mathrm{AD}$ prior to the network-based classification: the results of crossvalidation suggest that about $5 \%$ of individuals may be incorrectly classified with the CSF heuristic used here. ${ }^{29}$ Finally, while we assigned participants to clinical groups based on current clinical diagnostic practices, future studies could include A $\beta$-PET as an additional diagnostic procedure and covariate to examine robustness in classification analyses.

\section{AUTHOR CONTRIBUTIONS}

John D. Medaglia: study concept and design, writing of manuscript, data analysis. Weiyu Huang: machine learning analysis scripts and conceptualization. Santiago Segarra: machine learning analysis scripts and conceptualization. Christopher Olm: neuroimaging processing and manuscript editing. James Gee: neuroimaging processing. Murray Grossman: data collection, study concept, manuscript editing, obtained funding. Alejandro Ribeiro: supervision of machine learning analysis. Corey T. McMillan: manuscript editing, study concept. Danielle S. Bassett: study concept and design, manuscript editing, obtained funding.

\section{STUDY FUNDING}

Study was funded by a CBICA grant to Bassett, Gee, and Grossman. Prior data collection was funded by NIH grant AG017586, AG043503, Dana Foundation. D.S.B. also acknowledges support from the John D. and Catherine T. MacArthur Foundation and the Alfred P. Sloan Foundation. J.D.M. acknowledges support from NIH grant DP5OD021352. The content is solely the responsibility of the authors and does not necessarily represent the official views of any of the funding agencies.

\section{DISCLOSURE}

The authors report no disclosures relevant to the manuscript. Go to Neurology.org for full disclosures.

Received August 19, 2016. Accepted in final form March 10, 2017.

\section{REFERENCES}

1. Armstrong MJ, Litvan I, Lang AE, et al. Criteria for the diagnosis of corticobasal degeneration. Neurology 2013; 80:496-503.

2. McMillan CT, Brun C, Siddiqui S, et al. White matter imaging contributes to the multimodal diagnosis of frontotemporal lobar degeneration. Neurology 2012;78:1761-1768.

3. McMillan CT, Avants BB, Cook P, Ungar L, Trojanowski JQ, Grossman M. The power of neuroimaging biomarkers for screening frontotemporal dementia. Hum Brain Mapp 2014;35:4827-4840.

4. McMillan CT, Boyd C, Gross RG, Weinstein J, Firn K, Toledo JB. Multimodal imaging evidence of pathologymediated disease distribution in corticobasal syndrome. Neurology 2016;87:1227-1234.

5. van den Heuvel MP, Sporns O. Network hubs in the human brain. Trends Cogn Sci 2013;17:683-696.

6. Warren DE, Power JD, Bruss J, et al. Network measures predict neuropsychological outcome after brain injury. Proc Natl Acad Sci 2014;111:14247-14252.

7. Whitwell JL, Avula R, Senjem MS, et al. Gray and white matter water diffusion in the syndromic variants of frontotemporal dementia. Neurology 2010;74:1279-1287.

8. Newman MEJ. Networks: An Introduction. New York: Oxford University Press; 2010.

9. Rubinov M, Sporns O. Complex network measures of brain connectivity: uses and interpretations. NeuroImage 2010;52:1059-1069.

10. Suykens JAK, Vandewalle J. Least squares support vector machine classifiers. Neur Proc Lett 1999;9:293-300.

11. Rasmussen CE. Gaussian Processes for Machine Learning. Cambridge: MIT Press; 2006.

12. Arlot $\mathrm{S}$, Celisse A. A survey of cross-validation procedures for model selection. Stat Surv 2010;4:40-79.

13. Kuncheva LI, Whitaker CJ. Measures of diversity in classifier ensembles and their relationship with the ensemble accuracy. Mach Learn 2003;51:181-207.

14. Bonferroni CE. Teoria statistica delle classi e calcolo delle probabilita. Pub R Istitut Sup Sci Econ Commerc Fir 1936;8:1-62. 
15. McNemar Q. Note on the sampling error of the difference between correlated proportions or percentages. Psychometrika 1947;12:153-157.

16. Acosta-Cabronero J, Williams GB, Pengas G, Nestor PJ. Absolute diffusivities define the landscape of white matter degeneration in Alzheimer disease. Brain 2010;133:529-539.

17. Schroeter ML, Raczka K, Neumann J, Von Cramon DY. Neural networks in frontotemporal dementia: a meta-analysis. Neurobiol Aging 2008;29:418-426.

18. Lee SE, Rabinovici GD, Mayo MC, et al. Clinicopathological correlations in corticobasal degeneration. Ann Neurol 2011;70:327-340.

19. Khazaee A, Ebrahimzadeh A, Babajani-Feremi A. Application of pattern recognition and graph theoretical approaches to analysis of brain network in Alzheimer disease. J Med Imag Health Info 2015;5:1145-1155.

20. Stam CJ. Modern network science of neurological disorders. Nat Rev Neurosci 2014;15:683-695.

21. Dickson DW, Kouri N, Murray ME, Josephs KA. Neuropathology of frontotemporal lobar degeneration-tau (FTLD-tau). J Mol Neurosci 2011;45:384-389.

22. Medaglia JD, Lynall ME, Bassett DS. Cognitive network neuroscience. J Cogn Neurosci 2015;27:1471-1491.
23. Latora V, Marchiori M. Efficient behavior of small-world networks. Phys Rev Lett 2001;87:198701.

24. Vlooswijk MCG, Vaessen MJ, Jansen JFA, de Krom MCFTM, Majoie HJM, Hofman PAM, Backes WH. Loss of network efficiency associated with cognitive decline in chronic epilepsy. Neurology 2011;77: 938-944.

25. Kovacs GG. Invited review: neuropathology of tauopathies: principles and practice. Neuropathol Appl Neurobiol 2015;41:3-23.

26. Honey CJ, Thivierge JP, Sporns O. Can structure predict function in the human brain? NeuroImage 2010;52:766776.

27. Hagmann P, Kurant M, Gigandet X, Thiran P, Wedeen V, Meuli R. Mapping human whole-brain structural networks with diffusion MRI. PLoS One 2007;2:e597.

28. Dell'Acqua F, Catani M. Structural human brain networks: hot topics in diffusion tractography. Curr Opin Neurol 2012;25:375-383.

29. Irwin DJ, McMillan CT, Toledo JB, et al. Comparison of cerebrospinal fluid levels of tau and $\mathrm{Ab}$ in Alzheimer disease and frontotemporal degeneration using 2 analytical platforms. Arch Neurol 2012;69:1018-1025.

\section{Subspecialty Alerts by E-mail!}

Customize your online journal experience by signing up for e-mail alerts related to your subspecialty or area of interest. Access this free service by visiting Neurology.org/site/subscriptions/etoc.xhtml or click on the "E-mail Alerts" link on the home page. An extensive list of subspecialties, methods, and study design choices will be available for you to choose from—allowing you priority alerts to cutting-edge research in your field!

\section{Pि Neurology.org Offers Important Information to Patients and Their Families}

The Neurolog ${ }^{\circledR}$ Patient Page provides:

- A critical review of ground-breaking discoveries in neurologic research that are written especially for patients and their families

- Up-to-date patient information about many neurologic diseases

- Links to additional information resources for neurologic patients

All Neurology Patient Page articles can be easily downloaded and printed, and may be reproduced to distribute for educational purposes. Click on the 'Patients' link on the home page (Neurology.org) for a complete index of Patient Pages. 


\section{Neurology}

\section{Brain network efficiency is influenced by the pathologic source of corticobasal syndrome}

John D. Medaglia, Weiyu Huang, Santiago Segarra, et al.

Neurology 2017;89;1373-1381 Published Online before print August 4, 2017

DOI 10.1212/WNL.0000000000004324

This information is current as of August 4, 2017

\section{Updated Information \& Services}

Supplementary Material

\section{References}

Subspecialty Collections

\section{Permissions \& Licensing}

Reprints including high resolution figures, can be found at: http://n.neurology.org/content/89/13/1373.full

Supplementary material can be found at: http://n.neurology.org/content/suppl/2017/08/04/WNL.0000000000004 324.DC1

This article cites 27 articles, 6 of which you can access for free at: http://n.neurology.org/content/89/13/1373.full\#ref-list-1

This article, along with others on similar topics, appears in the following collection(s):

Alzheimer's disease

http://n.neurology.org/cgi/collection/alzheimers_disease Corticobasal degeneration

http://n.neurology.org/cgi/collection/corticobasal_degeneration

Frontotemporal dementia

http://n.neurology.org/cgi/collection/frontotemporal_dementia

MCI (mild cognitive impairment)

http://n.neurology.org/cgi/collection/mci_mild_cognitive_impairment

Information about reproducing this article in parts (figures,tables) or in its entirety can be found online at:

http://www.neurology.org/about/about_the_journal\#permissions

Information about ordering reprints can be found online:

http://n.neurology.org/subscribers/advertise

Neurology ${ }^{\circledR}$ is the official journal of the American Academy of Neurology. Published continuously since 1951, it is now a weekly with 48 issues per year. Copyright Copyright ( 2017 The Author(s). Published by Wolters Kluwer Health, Inc. on behalf of the American Academy of Neurology.. All rights reserved. Print ISSN: 0028-3878. Online ISSN: 1526-632X.

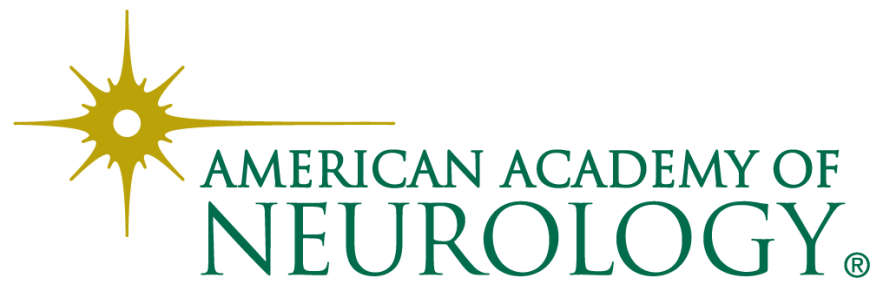

